SUPPLEMENTARY INFORMATION

\title{
Enhanced microfluidic sample homogeneity and improved antibody-based assay kinetics due to magnetic mixing
}

Eriola-Sophia Shanko, Lennard Ceelen, Ye Wang, Yoeri van de Burgt and Jaap den Toonder*

Microsystems Research Section, Department of Mechanical Engineering, and Institute for Complex Molecular Systems (ICMS), Eindhoven University of Technology, PO Box 513, 560oMB Eindhoven, The Netherlands.

Number of figures: 6 


\title{
Enhanced microfluidic sample homogeneity and improved antibody-based assay kinetics due to magnetic mixing
}

\author{
Eriola-Sophia Shanko, Lennard Ceelen, Ye Wang, Yoeri van de Burgt and Jaap den \\ Toonder*
}

Microsystems Research Section, Department of Mechanical Engineering, and Institute for Complex Molecular Systems (ICMS), Eindhoven University of Technology, PO Box 513, 560oMB Eindhoven, The Netherlands.

\section{MATERIALS AND METHODS}

\section{MAGNETIC BEADS AND ELECTROMAGNETS}

Two main components are required for magnetic bead mixing, namely (electro)magnets to generate a magnetic field and magnetic beads. A unique octopolar electromagnetic setup was previously constructed to offer $3 \mathrm{D}$ manipulation of magnetic beads by separately controlling opposing pairs of magnetic poles, as shown in Figure S1a. In this research, only the four poles in the vertical plane are used to generate an out of plane rotating magnetic field, relative to a microfluidic chamber placed in the center between of the poles (Figure S1b). The two pairs of poles are actuated in sinusoidal waveform with a 90 degrees phase difference, therefore, combined, a rotational field is generated. The amplitude of the field and the frequency are adjusted using a LabView platform.

Superparamagnetic iron oxide microbeads (SPIOM) $(\varnothing=10 \mu \mathrm{m}$, Micromer- M, Micromod, $\sim 6000$ beads $\left.\mu \mathrm{L}^{-1}\right)$ ) are used, coated with carboxylic group to prevent agglomeration. These are the same magnetic beads and concentration as in previous work. ${ }^{1}$

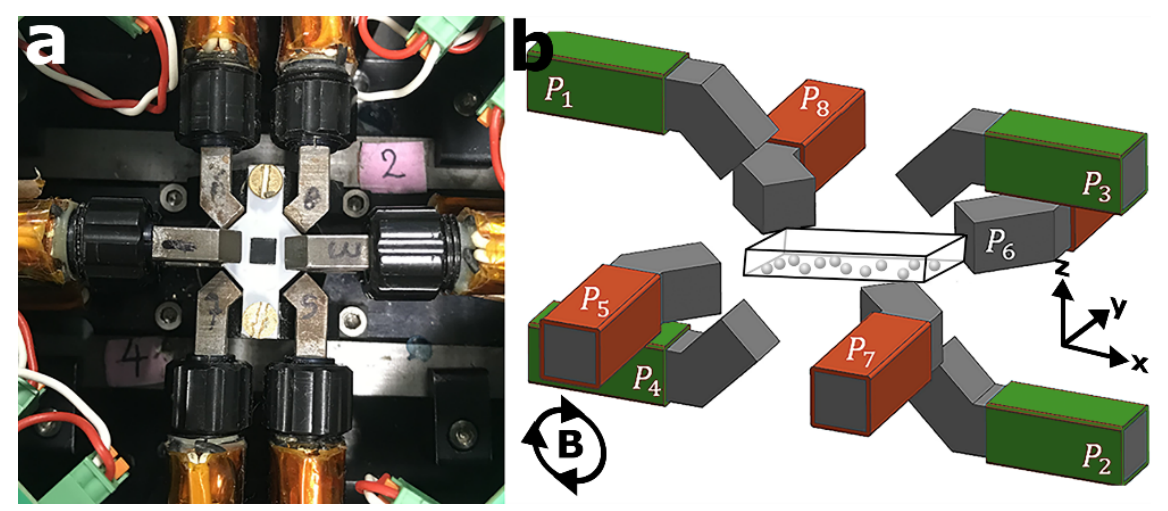

Figure S1: Magnetic setup and actuated poles. a) The octopolar electromagnetic setup (top view), with the microfluidic chamber in the center. Distance between opposite poles is $6 \mathrm{~mm}$. b) a schematic of the setup; only the green poles $\left(\mathrm{P}_{1}-\mathrm{P}_{4}\right)$ are actuated to induce a magnetic field rotating in the vertical plane.

A PMMA-based micro reaction chamber ( $4 \times 4 \mathrm{~mm}$ and $500 \mu \mathrm{m}$ high) is made by laminating PMMA sheets that are pre-cut using a laser cutter (VLS3.50, 40W CO2, Laser Universal Systems) and placed in the center of the electromagnetic setup (as also seen in Figure $\mathbf{S} \mathbf{1}$ ). The sample is then injected using a pipette with test fluids and covered with a thin glass slide. Finally, the whole setup is placed under a microscope (Leica DM40ooM) with a CMOS camera (Leica, DFC9oooGT). The visualization and quantification of the induced flow is realized with the addition of passive fluorescent particles $(\varnothing=4 \mu \mathrm{m}$, Fluoromax, Thermo Scientific). Fluid velocities are measured with $3 \mathrm{D}$ Particle Tracking Velocimetry techniques (see Supplementary Information S4). ${ }^{2}$ 


\section{HYSTERESIS LOOP OF THE MAGNETIC BEADS}

To properly perform theoretical analysis on our magnetic beads, characteristics of the magnetic properties (hysteresis loops for susceptibility determination) are obtained using an MPMS-3 superconducting quantum interference device (SQUID) from Quantum Design.

The sample of dried magnetic beads is placed in the SQUID magnetometer. The diamagnetic interference due to the presence of the plastic holder was noted and compensated for due to the low magnetic susceptibility of our beads. The resulting hysteresis loop of the 10 micrometer Micromod Micromer M- can be seen in Figure S2. Saturation is reached at an applied magnetic field magnitude of $B=2.5 \mathrm{~T}$ and saturation magnetization at $25 \mathrm{mT}$, and the slope of the curve at $\mathrm{o}$ is the dimensionless magnetic susceptibility, which is $\chi=0,04$ for the beads.



Figure S2: Hysteresis loop of the used magnetic elements - superparamagnetic beads.

\section{MIXING INDEX METHODS AND DETERMINATION}

For the mixing index measurements, $2 \mu \mathrm{L}$ of pluronic acid F-127 ( $1 \% \mathrm{w} \mathrm{v}-1)$ solution is first deposited in the chamber and left to dry at room temperature for 20 mins. This step ensures surface treatment of the PMMA chamber to avoid bubble formation when the chamber is covered with the glass slide. The chamber is then filled using a pipette with the SPIOM solution and covered with glass. The glass slide is slightly shifted to open a part in the top right corner of the chamber, where $1 \mu \mathrm{L}$ of a mixture of $75 \%$ glycerol (Sigma Aldrich, high purity) with concentrated fluorescent beads ( $\varnothing=2 \mu \mathrm{m}$, Fluoromax, Thermo Scientific) is injected using a pipette. Last, the glass slide is shifted back to fully cover the micro chamber, and the solution is left for approximately 11 minutes. The last step is taken to allow the two fluids with different viscosities to partially diffuse, and create a reproducible initial state for all the experiments.

The method of intensity of segregation is widely used for mixing performance assessments, ${ }^{2}$ and is chosen to quantify the Mixing Index (MI) which is used as a measure of mixing. The MI is defined as: ${ }^{3}$

$$
M I=\frac{1}{N} \frac{\sum_{N}\left(I_{k}-\frac{\sum_{N} I_{k}}{N}\right)^{2}}{\left(\frac{\sum_{N} I_{k}}{N}\right)^{2}}=\frac{\operatorname{var}(I)}{\bar{I}^{2}}
$$

In this equation, $\mathrm{I}_{\mathrm{k}}$ is the fluorescent intensity [0-255] at pixel $k$ and $(\bar{I})$ is the intensity averaged over all $\mathrm{N}$ pixels $(6.5 \mu \mathrm{m} \times 6.5 \mu \mathrm{m}$ pixel size, and $2048 \times 2048$ pixels). This indicates that in a perfectly mixed scenario $\mathrm{MI}=0$, whilst in a fully unmixed system $\mathrm{MI}=1$. In short, lower MI corresponds to better mixing. To obtain comparable results to the different mixing at different actuation conditions, the mean of a series of three 
experimental sets with similar initial MI states was collected. The RMI is a normalized version of the MI and is instead used as a mixing quantification method where every MI obtained in an image is divided over the MI of first attained image (initial state of experiment).

\section{LUMABS SENSOR}

The effect of increased kinetics due to induced SPIOM motion on a bioluminescent assay is investigated using the LUMABS sensor. ${ }^{4}$ LUMABS is based on bioluminescence resonance energy transfer (BRET) that currently allows the detection of antibodies against HIV1-p17, hemagglutinin (HA), and dengue virus type I, while assays for other markers are in development. ${ }^{5}$ LUMABS is a single-protein sensor that consists of the blue-light emitting luciferase NanoLuc connected via a semi flexible linker to the green fluorescent acceptor protein $\mathrm{mNeonGreen,} \mathrm{which} \mathrm{are} \mathrm{kept} \mathrm{close} \mathrm{together} \mathrm{using} \mathrm{helper} \mathrm{domains,} \mathrm{hence}$ emitting green light. Binding of an antibody to epitope sequences flanking the linker disrupts the interaction between the helper domains, resulting in a large decrease in BRET efficiency, hence blue emission is enhanced. Practically, the LUMABS is a combination of three components: the sensor, the substrate and the target antibody. The substrate acts as a fuel agent for the sensor, and thus their interaction is mandatory for the emission. The sensor and substrate are connected through a semi-flexible link; in the presence of an antibody this link breaks up resulting in blue emission (Figure $\mathbf{S}_{3}$ ). The change from green to blue emission depicts the antibody presence whilst the ratio of green over blue emission is the antibody quantification measure. ${ }^{5}$

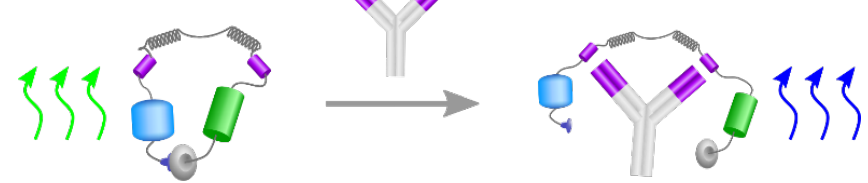

Figure S3: Schematic view of the LUMABS in the absence and presence of a corresponding antibody.

For these experiments, micro channels are added to the device to allow the solution flow into the micro reaction chamber. A mixture of glycerol and an $110 n \mathrm{M}$ sensor $(25 \%$ glycerol $)$ is printed on the microfluidic chamber floor via deposition and left to dry at room temperature for 2 hrs. The deposition entails manual pipetting of $2 \mu \mathrm{L}$ solution on the chamber floor. The liquid sample subsequently added is a mixture of antibody (CTX-2, various concentrations) and substrate (dilution of 1:15). The sample is injected using a pipette on the inlet $(\varnothing=1 \mathrm{~mm})$ of the microfluidic chip which is connected to the chamber with a microchannel (width $=200 \mu \mathrm{m}$ and height $=500 \mu \mathrm{m})$. The microfluidic chips are mounted in the octopolar electromagnetic setup and a commercially available camera (Canon EOS 8oD SLR) featuring a light sensitive macro lens (Canon EF 10omm $\mathrm{f} / 2.8 \mathrm{~L}$ ) is placed atop at working distance. The settings covering low light requirements are: 8", ISO 1280o, F/2.8.

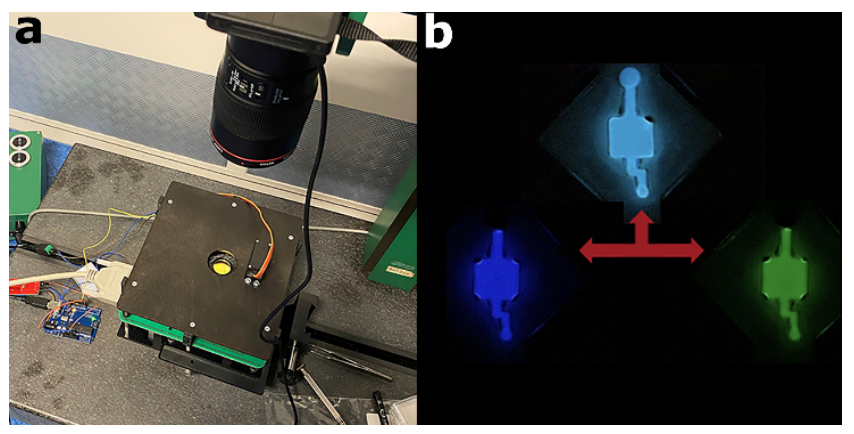

Figure S4: Camera setup for the biosensor experiments and resulting filtered images. a) The setup cover with the filters and the servo and its arrangement under the commercially available camera. b) The initial unfiltered image and the filtered images of only blue and only green.

To perfectly capture just the green emitting protein and the blue wavelengths without interferences, a lid with two bandpass filters (wavelength 450 and FWHM 10onm and wavelength 560 and FWHM 10onm (Omega filters)) is constructed. A servo connected to an Arduino which in turn is controlled via a custom 
script is alternating these filters (Figure $\mathbf{S}_{\mathbf{4}} \mathbf{a}$ ). In this way only blue or green wavelengths (Figure $\mathbf{S}_{\mathbf{4}} \mathbf{b}$ ) can be captured.

Images are taken every 10 seconds between the green and blue and there is an additional waiting time of 10 seconds after every loop. Thus, within a minute, two couples of images are obtained. Last, a magnetic mixing protocol is initiated for the effect of magnetic mixing to the sensor's sensitivity and time to readout.

\section{FORCES AT PLAY IN MAGNETIC BEADS' MOTION IN THE FLUID}

\section{DIPOLE FORCES:}

The expression for the magnetic interaction force $\vec{F}_{i}^{m i}$ between the $\mathrm{i}^{\text {th }}$ superparamagnetic particle with the rest of the $(N-1)$ particles is:

$$
\vec{F}_{i}^{m i}=\frac{3 \mu_{0}}{4 \pi} \sum_{j=1, j \neq i}^{N} \frac{m_{p i} m_{p j}}{r_{i j}^{4}}\left[\left(1-5\left(\widehat{\vec{m}} \cdot \widehat{\widehat{r_{l j}}}\right)^{2}\right) \widehat{\widehat{r_{l j}}}+2\left(\widehat{\vec{m}} \cdot \widehat{\overrightarrow{r_{l j}}}\right) \widehat{\vec{m}}\right]
$$

where $m_{p i}$ is the strength of the dipole moment of the ith particle, $\widehat{\vec{m}}$ is the unit vector of the magnetization, $r_{i j}$ the disctance between the centers of the ith and jth particles, and $\widehat{\overrightarrow{r_{l j}}}$ the unit vector of the corresponding two-particle chain axis. ${ }^{6}$

\section{MAGNETIC AND VISCOUS DRAG TORQUE ON A SUPERPARAMAGNETIC PARTICLE CHAIN:}

A superparamagnetic particle chain in a rotating magnetic field experiences a magnetic torque $\vec{\tau}_{\text {mag }}=$ $\vec{m}_{c} \times \vec{B}$ that tends to align the chain to the magnetic field $\vec{B}$. An explicit expression for the magnitude of the torque for an $\mathrm{N}$-bead chain is:

$$
\tau_{m a g}=\frac{3 \mu_{0} m^{2}(N-1)}{4 \pi(2 R)^{3}} \sin (2 \alpha)
$$

where $\alpha$ is the angle (i.e. phase lag) between the chain-axis and the magnetic field. ${ }^{7}$

An N-particle chain rotating at angular velocity $\omega=d \theta / d t$ in a fluid with viscosity $\eta$ experience a viscous drag torque the magnitude of which can be explicitly approximated by: ${ }^{7}$

$$
\tau_{\text {drag }}=\frac{8 \pi R^{3}}{3} \frac{N^{3}}{\ln \left(\frac{N}{2}\right)+\frac{2.4}{N}} \eta \omega
$$




\section{MOVIES OF THE COLLECTIVE MAGNETIC BEAD MOTION}

\section{MAGNETIC BEAD BEHAVIOR AT 25mT AND AT VARIOUS FREQUENCIES}

In this section the collective magnetic bead motion at $25 \mathrm{mT}$ vertical magnetic rotation (where only the four vertical poles -P1-4 (Figure. Sib)- are actuated), at different rotation frequencies. The videos display the top view of the $4 \mathrm{X} 4 \mathrm{~mm}$ microfluidic chamber (i.e. the xy-plane in Figure. Sib). Magnetic Particle Swarming is observed at actuation frequencies between $20 \mathrm{~Hz}$ and $50 \mathrm{~Hz}$, here visible in the $30 \mathrm{~Hz}$ results.

Movie S3.1. Global magnetic bead behavior in a clockwise vertical rotating magnetic field at $1 \mathrm{~Hz}, 25 \mathrm{mT}$ as recorded real-time. The magnetic beads are forming long chains and they are rotating around their long axis (local rotation) and agglomerate towards the right poles $\left(\mathrm{P}_{2}\right.$ and $\left.\mathrm{P}_{3}\right)$ (global motion). They stay at one side of the chamber forming an island of beads/chains. A pulsatile notion is also noticed. QR code corresponding to the video:

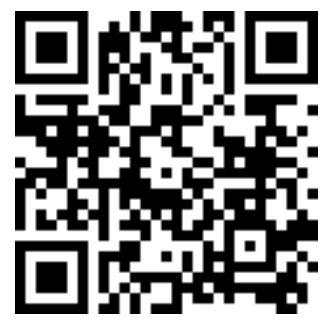

Movie S3.2.: Global magnetic bead behavior in a clockwise vertical rotating magnetic field at $10 \mathrm{~Hz}, 25 \mathrm{mT}$ as recorded real-time. The magnetic beads are forming chains and they are rotating around their long axis (local rotation) and move towards the right poles $\left(\mathrm{P}_{2}\right.$ and $\left.\mathrm{P}_{3}\right)$ (global motion). After a while, some magnetic beads, chains and clusters, are moving to the opposite side $\left(\mathrm{P}_{1}\right.$ and $\left.\mathrm{P}_{4}\right)$. A pulsatile notion is also noticed. The $10 \mathrm{~Hz}$ is at the boundary of Magnetic Particle Swarming (MPS) formation and island. QR code corresponding to the video:

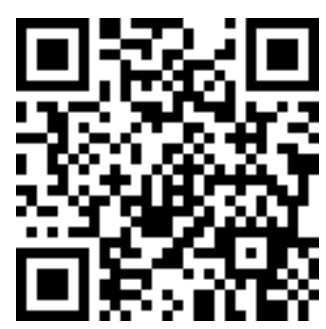

Movie S3.3: Global magnetic bead behavior in a clockwise vertical rotating magnetic field at 3oHz, 25mT as recorded real-time. The magnetic beads are forming short chains and clusters and they are rotating around themselves (local rotation) but they are also exhibiting global motion towards the right poles $\left(\mathrm{P}_{2}\right.$ and $\left.\mathrm{P}_{3}\right)$ (global motion). Soon, the magnetic beads, chains and clusters, are moving to the opposite side $\left(\mathrm{P}_{1}\right.$ and $\left.\mathrm{P}_{4}\right)$ and eventually they are forming a stable global circulatory motion in the microfluidic chamber; this is the Magnetic Particle Swarming (MPS). QR code corresponding to the video:

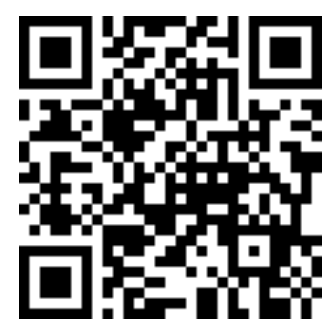

Movie S3.4: Global magnetic bead behavior in a clockwise vertical rotating magnetic field at $60 \mathrm{~Hz}, 25 \mathrm{mT}$ as recorded real-time. The magnetic beads are forming short chains and clusters and they are rotating around themselves (local rotation) but they remain in an island at the right poles $\left(\mathrm{P}_{2}\right.$ and $\left.\mathrm{P}_{3}\right)$ (no global motion). The $6 \mathrm{oHz}$ is at the boundary of Magnetic Particle Swarming (MPS) formation and island. QR code corresponding to the video: 




\section{SIDE VIEW OF THE MAGNETIC BEAD BEHAVIOR DURING THE MAGNETIC PARTICLE SWARMING}

To verify that the formation of the stable circulatory SPIOM motion is due to micro crawling we take advantage of the full capabilities of the electromagnetic setup. Since it is difficult to visualize the SPIOM dynamics on the side view of the micro chamber, we rotate the chamber by $90^{\circ}$ and apply a horizontal magnetic field.

Movie S3.5: Side view of the magnetic particle swarming at 35mT, 30Hz. QR code corresponding to the video:

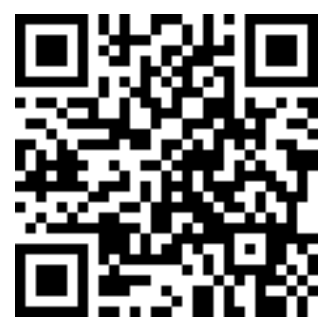

\section{LOCAL DYNAMICS OF PARTICLE-PARTICLE INTERACTIONS.}

On a local scale, the particle motion is quite dynamic, with continuous breakup and reformation of chains and clusters. These local effects also contribute to mixing.

Movie S3.6: Local dynamics in particle-particle interactions at 35mT, $1 \mathrm{~Hz}$. QR code corresponding to the video:



Movie S3.7: Local dynamics in particle-particle interactions and chain break up and reformation at 35mT, 3oHz. QR code corresponding to the video:






\section{EFFECT OF INERTIA ON MAGNETIC BEAD MOTION}

To explore whether inertia plays a role in our experiments, we switched the magnetic field off during the Magnetic Particle Swarming (MPS) motion ( $30 \mathrm{~Hz}, 35 \mathrm{mT}$ ). As expected in a situation where inertia does not play a role, the magnetic beads stopped moving immediately and started sedimenting due to gravity.

Movie S3.8: The sedimentation of the magnetic beads as soon as the magnetic field is switched off during Magnetic Particle Swarming at $35 \mathrm{mT}$ and $30 \mathrm{~Hz}$. QR code corresponding to the video:

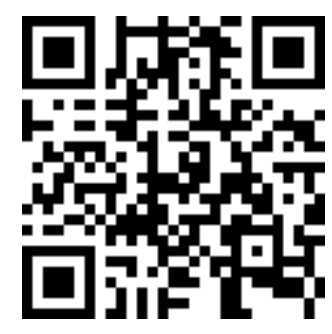

\section{4. $\quad 3 D$ PTV AND TRAJECTORIES OF FLUID TRACERS}

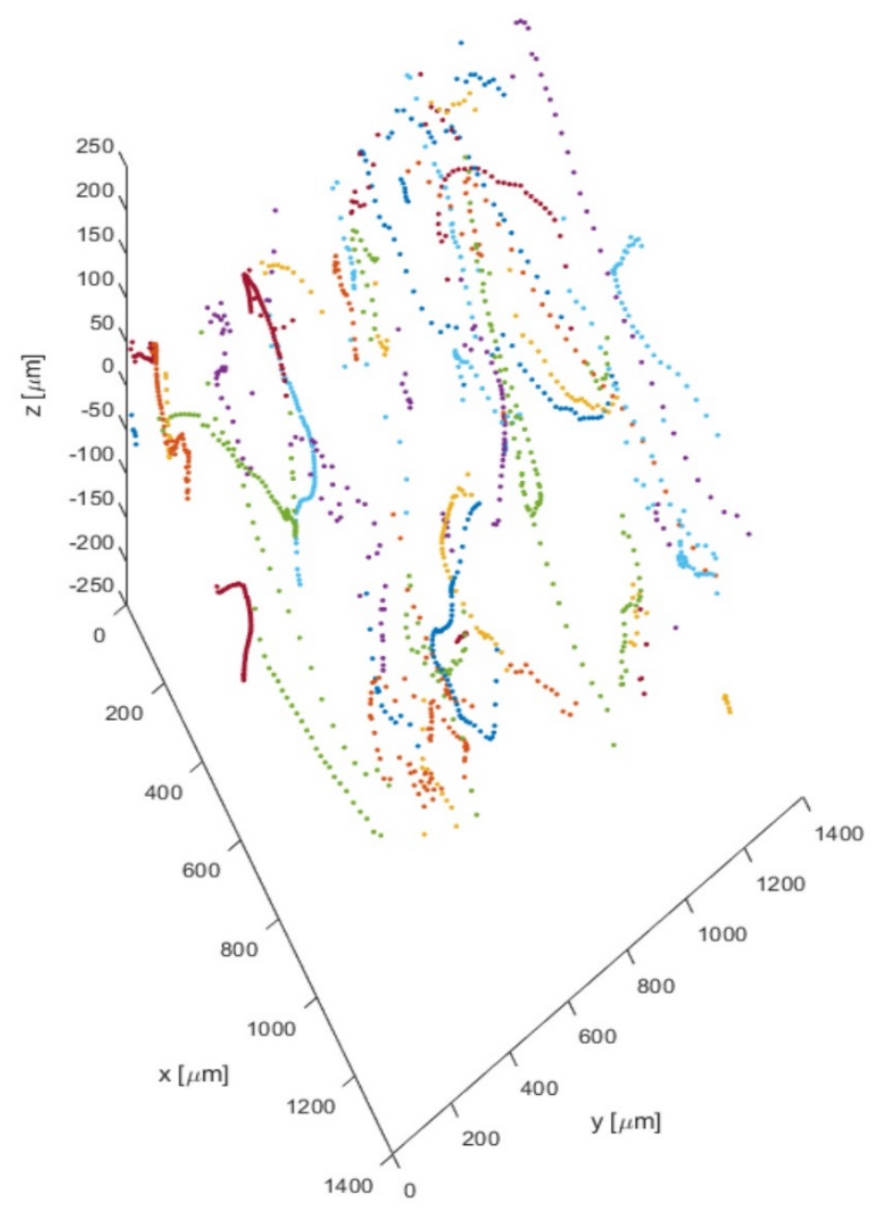

Figure $S_{5}$ : Trajectories of passive fluid tracers for the determination of fluid profiles and velocities in a $3 \mathrm{D}$ manner. The $3 \mathrm{D}$ particle tracking velocimetry was performed based on the general defocusing method. For this, a set of pre calibrated images of the fluorescent tracers was used as a reference image stack to determine the depth of the tracer particles within the volume. ${ }^{2}$ 


\section{MIXING EXPERIMENTS ANALYSIS}

The mixing assessment was based on the method of segregation. For this, a clear interface between two solutions with different colors (black and white) was needed. A solution of magnetic beads in water was first used to fill the rectangular chamber. Next, a $1 \mu \mathrm{L}$ of a more viscous solution seeded with fluorescent particles in $75 \%$ glycerol, was injected into the corner of the chamber manually through pipetting. In the fluorescence mode (Leica DM4oooM, Germany)) we could visualize the two separated fluids. To avoid including mixing through convection due to the pressure applied from the pipette upon the injection of the fluorescent solution, in our analysis, we first performed a long experiment to see when a clear interface was reached. During the long experiments we realized that it required approximately 11 minutes until convection due to the in injection of the solution was reduced, and from that point on a reproducible state based only on diffusion occurred. This became our initial waiting time for all experiments. In this particular experiment a comparison between the waiting time of 11 minutes and no waiting time is depicted.

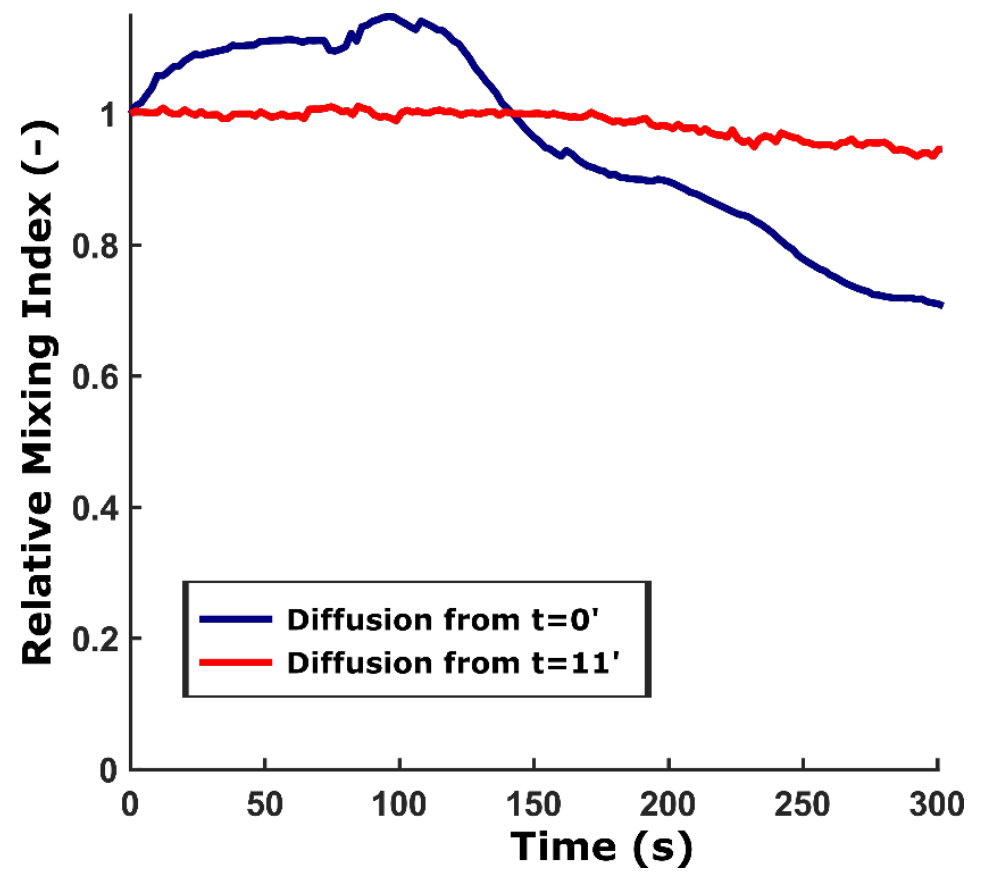

Figure S6: The relative mixing index in waiting time and lack thereof for the initial state

This procedure benefitted two purposes. First, the initial state is reproducible, thus experiments can be compared between them and second, during the no mixing state (control/diffusion), influences due to the initial pressure applied from the pipette when injecting the solution were offset. Last, an example of the videos are seen below.

Movie S5.1. Real-time mixing efficiency evolution over time on control (no mixing/ diffusion). Movie sped up and adjusted brightness and contrast. QR code corresponding to the video:

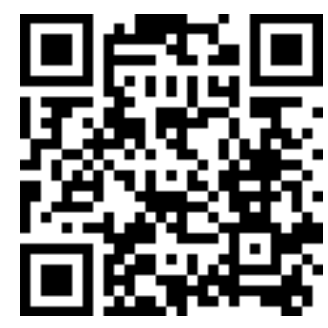

Movie S5.2: Mixing efficiency evolution over time on magnetic bead behavior at 15mT, 30Hz. Movie sped up and adjusted brightness. QR code corresponding to the video: 


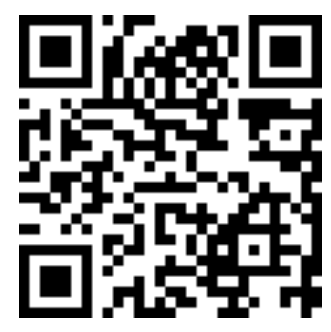

Movie S5.2: Real-time mixing efficiency evolution over time on magnetic bead behavior at $35 \mathrm{mT} 30 \mathrm{~Hz}$. Movie sped up and adjusted brightness and contrast. QR code corresponding to the video:



\section{MOVIES OF THE LUMABS}

The results of the sensor behavior are seen in this section. The LUMABS sensor is deposited on the floor of a microfluidic chamber, and the sample containing the magnetic beads, the target protein and substrate molecules are entered into the chamber. Three different states are applied: no actuation / mixing, actuation at $15 \mathrm{mT}$ in which poor mixing and no Magnetic particle Swarming is observed, and actuation at $35 \mathrm{mT}$ with better mixing and Magnetic Particle Swarming. Inhomogeneity was reduced by mixing and the change in color is faster and larger in the mixed states. The magnetic rotational frequency is $30 \mathrm{~Hz}$, the antibody concentration is $1,1 \mu \mathrm{M}$ and the sensor concentration is $110 \mathrm{MM}$. Scale bar is $4 \mathrm{~mm}$.

Movie S6.1: Sensor behavior in the non-mixed state in a 24 minute protocol of antibody concentration at 1, $1 \mu \mathrm{M}$ in a 24 minute protocol. (Movie sped up). QR code corresponding to the video. Left: Blue, Right: Green.
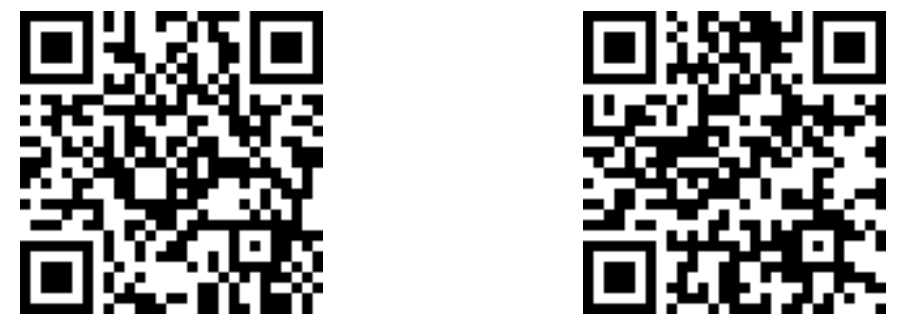

Movie S6.2: Sensor behavior in the mixing state occurred at $15 \mathrm{mT}, 30 \mathrm{~Hz}$ in a 24 minute protocol of antibody concentration at 1, $1 \mu \mathrm{M}$. (Movie sped up). QR code corresponding to the video: Left: Blue, Right: Green.
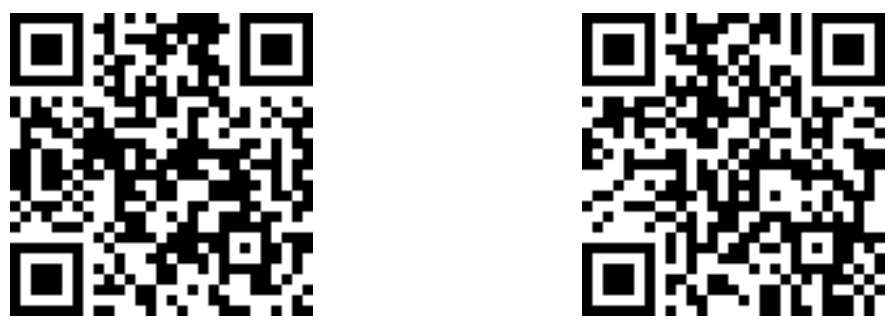

Movie S6.3: Sensor behavior in the mixing state occurring at $35 \mathrm{mT}, 30 \mathrm{~Hz}$ with the Magnetic Particle Swarming (MPS) in a 24 minute protocol of antibody concentration at $1,1 \mu \mathrm{M}$. (Movie sped up). QR code corresponding to the video: Left: Blue, Right: Green. 

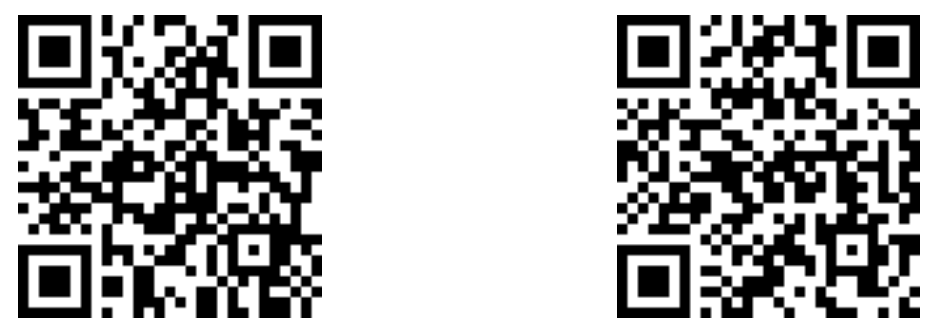

\section{REFERENCES:}

1. Gao, Y.; Beeren, J.; van Reenen, A.; Hulsen, M. A.; de Jong, A. M.; Pring, M. W. J.; den Toonder, J. M. J.. Strong vortical flows generated by the collective motion of magnetic particle chains rotating in a fluid cell. Lab Chip 2015, 15, 351-36o.

2. Barnkob, R.; Kähler, C. J.; Rossi, M. General defocusing particle tracking. Lab Chip 2015, 15, 35563560 .

3. Kim, S. D; Song, S. W.; Oh, D. Y.; Lee, A. C.; Koo, J. W.; Kang, T., Kim, M. C.; Lee, C., Jeong, Y.; Jeong, H. Y.; Lee, D.; Cho, S.; Kwon, S.; Kim, J. . Microspinning: local surface mixing via rotation of magnetic microparticles for efficient small-volume bioassays. Micromachines 2020, 11 (2), 175.

4. Van Rosmalen, M.; Ni, Y.; Vervoort, D. F. M.; Arts, R.; Ludwig, S. K. J.; Merkx, M. . Dual-color bioluminescent sensor proteins for therapeutic drug monitoring of antitumor antibodies. Anal. Chem. 2018, 90, 3592-3599.

5. Arts, R.; den Hartog, I.; Zijlema, S. E.; Thijssen, V.; van der Beelen, S. H. E.; Merkx, M. Detection of Antibodies in Blood Plasma Using Bioluminescent Sensor Proteins and a Smartphone. Anal. Chem. 2016, $88,4525-4532$.

6. Gao, Y.; van Reenen A.; Hulsen M. A.; de Jong A. M.; Prins M. W. J; den Toonder J. M. J. Disaggregation of microparticle clusters by induced magnetic dipole-dipole repulsion near a surface. Lab Chip 2013, 13, 1394.

7. Gao, Y.; Hulsen, M. A.; Kang, T. G.; den Toonder, J. M. J. Numerical and experimental study of a rotating magnetic particle chain in a viscous fluid. Phys. Rev. E 2012, 041503, 1-11. 